\begin{tabular}{|c|c|c|}
\hline \multirow{3}{*}{$\begin{array}{r}\text { Case Reports in } \\
\text { Gastroenterology }\end{array}$} & \multirow{2}{*}{\multicolumn{2}{|c|}{ Case Rep Gastroenterol 2015;9:137-141 }} \\
\hline & & \\
\hline & $\begin{array}{l}\text { DOI: } 10.1159 / 000382073 \\
\text { Publisnea onnine: Tviay } 7,2015\end{array}$ & $\begin{array}{l}\text { ( } 2015 \text { S. Karger AG, Basel } \\
1662-0631 / 15 / 0092-0137 \$ 39.50 / 0 \\
\text { www.karger.com/crg }\end{array}$ \\
\hline & \multicolumn{2}{|c|}{$\begin{array}{l}\text { This is an Open Access article licensed under the terms of the Creative Common } \\
\text { Attribution-NonCommercial } 3.0 \text { Unported license (CC BY-NC) (www.karger.com/OA } \\
\text { license), applicable to the online version of the article only. Distribution permitted for non } \\
\text { commercial purposes only. }\end{array}$} \\
\hline
\end{tabular}

\title{
Factitious Disorder in Crohn's Disease: Recurrent Pancytopenia Caused by Surreptitious Ingestion of 6-Mercaptopurine
}

\author{
Allon Kahn ${ }^{a}$ Erika S. Boroff ${ }^{b} \quad$ Kari A. Martin ${ }^{c} \quad$ Donald W. Northfelt ${ }^{d}$ \\ Russell I. Heigh ${ }^{\mathrm{b}}$ \\ ${ }^{\mathrm{a}}$ Department of Medicine, ${ }^{\mathrm{b}}$ Division of Gastroenterology and Hepatology, ${ }^{\mathrm{c}}$ Department of \\ Psychiatry and ${ }^{\mathrm{d}}$ Division of Hematology and Oncology, Mayo Clinic, Phoenix, Ariz., USA
}

\section{Key Words}

Factitious disorder · Medication · Complication - Inflammatory bowel disease · Intentional ·

Dyserythropoiesis · Anemia · Thrombocytopenia $\cdot$ Leukopenia

\begin{abstract}
Factitious disorder is a rare psychiatric illness characterized by the willful and deceptive induction of illness for the purpose of assuming the sick role. It presents a substantial diagnostic challenge, as patients often go to great lengths to conceal their deception. Accordingly, its presence in the full spectrum of gastrointestinal diseases is likely underappreciated. While factitious gastrointestinal bleeding, abdominal pain and diarrhea are relatively common, factitious non-gastrointestinal symptoms in the setting of gastrointestinal illness have been infrequently reported. We present the case of a patient with Crohn's disease with recurrent pancytopenia attributed to the surreptitious ingestion of 6-mercaptopurine. In patients with possible access to immunomodulatory drugs, a high suspicion for and early identification of factitious disorder may improve patient outcomes and avoid invasive and costly diagnostic evaluations.

(C) 2015 S. Karger AG, Basel
\end{abstract}

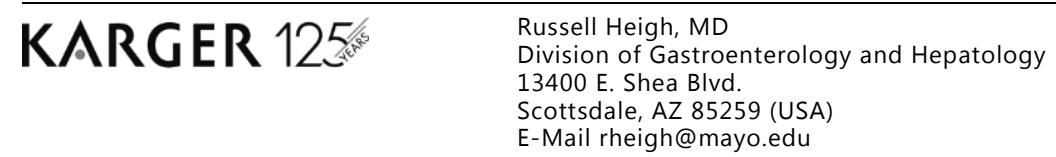


Kahn et al.: Factitious Disorder in Crohn's Disease: Recurrent Pancytopenia Caused by Surreptitious Ingestion of 6-Mercaptopurine

\section{Introduction}

Factitious disorder (FD) is defined in the 5th edition of the Diagnostic and Statistical Manual for Mental Disorders as the falsification of physical or psychological signs or symptoms, or induction of injury or disease, associated with identified deception [1]. In the spectrum of feigned illness, FD is distinguished by the absence of an overt external reward. In contrast, malingering is the intentional induction or falsification of illness for the acquisition of secondary gain [2]. Clinical manifestations of FD are variable and categorized as either predominantly psychiatric or physical. Existing reports in the gastroenterology literature have been characteristically limited to factitious abdominal pain, diarrhea and gastrointestinal bleeding (GIB) [3-5]. Conversely, non-gastrointestinal presenting manifestations of FD in the setting of gastrointestinal disorders, such as inflammatory bowel disease (IBD), have been infrequently reported. We report a case of recurrent pancytopenia in a patient with Crohn's disease surreptitiously ingesting 6-mercaptopurine (6-MP).

\section{Case Presentation}

A 50-year-old female with an 8-year history of fistulizing Crohn's disease and active recurrent perirectal abscesses presented to the outpatient clinic for initial consultation. She had undergone a diverting colostomy elsewhere 2 years prior to consultation and reported a history of greater than 30 perianal surgical procedures. Her medical history also consisted of vitamin D deficiency, peripheral neuropathy, osteopenia and obstructive lung disease related to ongoing tobacco use. She reported unsuccessful treatment with 5-aminosalicylic acid agents, methotrexate, infliximab, adalimumab and certolizumab pegol.

The patient was started on $75 \mathrm{mg}$ of 6-MP daily, however severe abdominal pain and active perianal disease persisted. Thiopurine metabolite testing for thioguanine nucleotide (TGN) and methyl mercaptopurine nucleotide (MMPN) revealed low levels and the dose was increased to $100 \mathrm{mg}$ daily. Six weeks later she presented with worsening abdominal pain. Laboratory testing demonstrated elevated lipase levels (4 times upper limit of normal) and the patient was told to discontinue 6-MP indefinitely, both by telephone and at a face-to-face follow-up visit, because of probable drug-induced pancreatitis

Two months later, the patient presented to the emergency department with a 3-day history of progressive headache, fatigue and a spreading erythematous rash. Laboratory studies demonstrated pancytopenia, with hemoglobin of 8.3, mean corpuscular volume of 112 , leukocyte count of 1.7, absolute neutrophil count of 0.42 and platelet count of 90 . A hematology consultation was obtained and her prior use of 6-MP dosing, followed by cessation, was specifically noted. The pancytopenia was attributed to residual effect of long-term 6-MP use and she was closely monitored, with counts progressively recovering throughout the remainder of her hospitalization.

Infliximab was reintroduced, and laboratory tests showed normalization of blood counts through the 4-month follow-up visit. However, she subsequently returned to the emergency department 8 days later with abdominal pain and increased ostomy output. Pancytopenia was again present with hemoglobin, leukocyte count and platelet count of 7.2, 2.1 and 42, respectively. Long-term immune-suppressive therapy was suspected as the etiology of the pancytopenia. Bone marrow biopsy showed hypocellularity, panhypoplasia and mild dyserythropoiesis. Further analysis demonstrated diploid XX cytogenetics and normal flow cytometric analysis, with no clonal or aberrant leukocyte population identified and no blast 
Kahn et al.: Factitious Disorder in Crohn's Disease: Recurrent Pancytopenia Caused by Surreptitious Ingestion of 6-Mercaptopurine

proliferation. She underwent transfusion of numerous blood products and ultimately was started on filgrastim. Her counts improved and she was discharged.

The patient was readmitted twice over the next 5 months with recurrent pancytopenia. A repeat bone marrow biopsy remained non-diagnostic. Again, she was transfused and given colony-stimulating factors as well as a prednisone taper and her counts improved. Several months later, she was readmitted for pancytopenia, oral ulcers and increased ostomy output. Out of concern for her repeated admissions with no identified cause, thiopurine metabolite testing was repeated and demonstrated TGN and MMPN levels of 385 (therapeutic window 230-400 pmol) and 12,417 (target $<5,700$ pmol to minimize toxicity), respectively, consistent with drug being present in the body and with MMPN levels predisposing toward toxicity. In the course of her evaluation, her community pharmacist contacted her outpatient providers and indicated that a refill of 6-MP had been dispensed 1 month prior to admission. The patient denied refilling the old prescription and indicated she did not take the 6-MP. The difficult situation was directly discussed with the patient and she again denied taking the 6-MP. She was specifically advised to not use this medication as it was likely responsible for prior pancreatitis and recurrent pancytopenia. Budesonide was initiated and all hematologic abnormalities resolved by 3 months. The patient subsequently canceled appointments at our institution and has been out of contact for 6 months.

After the pancreatitis attributed to 6-MP in January 2013, the patient was hospitalized 8 times during the time of this report extending to June 2014. Four of these admissions were related to pancytopenia. She had multiple consultations with gastroenterology, hematology, colorectal surgery and single consultations with infectious disease, rheumatology, dermatology, endocrinology and pulmonary medicine. At each hospital discharge, a written list of prescriptions was specifically reviewed with and provided to the patient. Medication reconciliation was performed at each outpatient visit, and her list, from which 6-MP was absent, was confirmed on every occasion.

\section{Discussion}

FD was first described in the second century by Claudius Galen, a Roman physician who reported feigned medical illness, including factitious GIB. The British physician Hector Gavin coined the term 'factitious disorder' in 1843 when he published a systematic account of feigned illness in soldiers and seamen [5]. It was not until 1951 that Dr. Richard Asher coined the widely recognized term 'Munchausen syndrome' for the most severe variety, in which the deceptive induction of illness becomes the central preoccupation of the patient [6]. A large retrospective cohort study of FD patients spanning 21 years showed a Caucasian female preponderance, with an average age of 30.7 years [7]. Modern estimates suggest that FD is present in approximately $1.3 \%$ of all hospitalized patients [8]. However, given the central role of deception in the classification of the disorder, it is likely grossly underdiagnosed and therefore underestimated.

To our knowledge, this is the first reported case of FD presenting without gastrointestinal manifestations in a patient with IBD. Outside of the gastroenterology literature, pancytopenia is a relatively common presenting manifestation of FD. In contrast, the existing gastroenterology literature regarding FD has been limited to several case reports of factitious GIB, diarrhea and abdominal pain. The mode of disease induction in cases of GIB varied greatly and included autophlebotomy and oral ingestion of blood [9], administration of blood enemas and injection of blood into the abdomen to simulate extravasation [10], swallowing razor blades [11], and disconnection and infusion of radioisotope-tagged red blood cells 
Kahn et al.: Factitious Disorder in Crohn's Disease: Recurrent Pancytopenia Caused by Surreptitious Ingestion of 6-Mercaptopurine

directly into the rectum during a scintigraphy study [12]. All reported patients had undergone extensive diagnostic testing without identified cause, including conventional radiography, endoscopy and scintigraphy.

Factitious diarrhea is also well described and often occurs in the setting of surreptitious laxative ingestion, whether for obtaining medical care or other secondary purposes, such as weight loss [5]. In some instances of gastrointestinal FD, patients attributed their symptoms to exacerbations of IBD, either diagnosed by themselves or by other physicians whose identities could not be verified [4, 12]. A high prevalence of chronic abdominal pain amongst patients with gastrointestinal disorders, including IBD, has led to anecdotal reports of factitious pain leading to hospitalization. Narcotic drug dependence is also noted to be highly prevalent in IBD, however feigned illness for obtaining medication would be classified as malingering for secondary gain.

While no systematic analysis of FD in IBD has been undertaken, there is growing recognition that patients with IBD face unique psychosocial challenges. Studies of pediatric IBD patients have demonstrated marked difficulty with social assimilation and resulting isolation, leading to increased absenteeism and refusal to participate in extracurricular activities [13]. Examinations of the connection between psychiatric illness and IBD have been fraught with controversy due to methodological heterogeneity. However, they have consistently demonstrated high rates of concurrent depression and anxiety at all ages, beyond what is seen in healthy controls and demographically matched patients with other chronic diseases [14]. Concomitant psychiatric illness may also place patients at risk of poor outcomes, as they experience more frequent disease relapse [15]. Examinations of the connection between self-perceived stress, irrespective of psychiatric illness, and IBD disease activity have yielded contradictory findings.

The diagnosis of FD is notoriously difficult and requires both a high index of suspicion and a systematic approach to exclusion of medical etiology. While not diagnostic, there are several characteristics that suggest the presence of feigned illness when medical causes have been sufficiently evaluated. These include treatment at multiple hospitals and/or clinics, an inordinate number of diagnostic tests without disease identification, eager agreement for invasive testing, unwillingness to provide prior medical records, history of employment or studies within health-related fields, symptom magnitude inconsistent with objective findings, opposition to psychiatric assessment and atypical disease course [3]. Of these, the atypical intermittent nature of our patient's disease course and the similarity to thioguanine toxicity was the aspect which drew suspicion and led to her diagnosis.

This case illustrates several clinical points. Patients with IBD often have comorbidities that include psychiatric illness, and frequently possess an in-depth knowledge of the medical system by virtue of their life experiences. For an inappropriately motivated patient, access to potent immunomodulatory agents provides an opportunity to experience problems unexpected by a treating team. Clinical suspicion of factitious illness is critical to management, as significant morbidity and even mortality may ensue. Early identification may ultimately improve patient outcomes. In spite of examination by multiple providers with elaborate processes to ensure reconciliation of medications prescribed and medications being actually taken, a determined patient may be taking substances unknown to the treating medical team. Unfortunately, as was seen in this case, patients confronted with evidence of their deception may disconnect entirely from their providers and seek a new health care system. 
Kahn et al.: Factitious Disorder in Crohn's Disease: Recurrent Pancytopenia Caused by Surreptitious Ingestion of 6-Mercaptopurine

\section{References}

1 American Psychiatric Association: Diagnostic and Statistical Manual of Mental Disorders, Fifth Edition. Arlington, American Psychiatric Association, 2013.

2 Sutherland AJ, Rodin GM: Factitious disorders in a general hospital setting: clinical features and a review of the literature. Psychosomatics 1990;31:392-399.

3 Bass C, Halligan P: Factitious disorders and malingering: challenges for clinical assessment and management. Lancet 2014;383:1422-1432.

4 Huffman JC, Stern TA: The diagnosis and treatment of Munchausen's syndrome. Gen Hosp Psychiatry 2003;25:358-363.

5 Savino AC, Fordtran JS: Factitious disease: clinical lessons from case studies at Baylor University Medical Center. Proc (Bayl Univ Med Cent) 2006;19:195-208.

6 Asher R: Munchausen's syndrome. Lancet 1951;1:339-341.

7 Krahn LE, Li H, O'Connor MK: Patients who strive to be ill: factitious disorder with physical symptoms. Am J Psychiatry 2003;160:1163-1168.

-8 Fliege H, Grimm A, Eckhardt-Henn A, Gieler U, Martin K, Klapp BF: Frequency of ICD-10 factitious disorder: survey of senior hospital consultants and physicians in private practice. Psychosomatics 2007;48:60-64.

$\$ 9$ McIntyre AS, Kamm MA: A case of factitious colonic bleeding. J R Soc Med 1990;83:465-466.

10 Bakkers JT, Crobach LF, Pauwels EK: Factitious gastrointestinal bleeding. J Nucl Med 1985;26:666-667.

11 Barkin JA, Biagini TM, Barkin JS: Factitious disorder as a cause of gastrointestinal bleeding: use of a gastroenterologist's 'secondary survey'. Am J Gastroenterol 2013;108:456-458.

12 Foga MM, Leslie WD: Gastrointestinal bleeding, Munchausen style. Clin Nucl Med 2003;28:330-331.

13 Desir B, Seidman EG: Transitioning the paediatric IBD patient to adult care. Best Pract Res Clin Gastroenterol 2003;17:197-212.

14 Engstrom I: Mental health and psychological functioning in children and adolescents with inflammatory bowel disease: a comparison with children having other chronic illnesses and with healthy children. J Child Psychol Psychiatry 1992;33:563-582.

-15 Mittermaier C, Dejaco C, Waldhoer T, Oefferlbauer-Ernst A, Miehsler W, Beier M, Tillinger W, Gangl A, Moser G: Impact of depressive mood on relapse in patients with inflammatory bowel disease: a prospective 18-month follow-up study. Psychosom Med 2004;66:79-84. 